\title{
Partial splenectomy followed by total splenectomy in thalassemia
}

\author{
Abu Saleh Md. Oli Ullah, Bhupal Chandra Barman and Md. Ruhul Amin
}

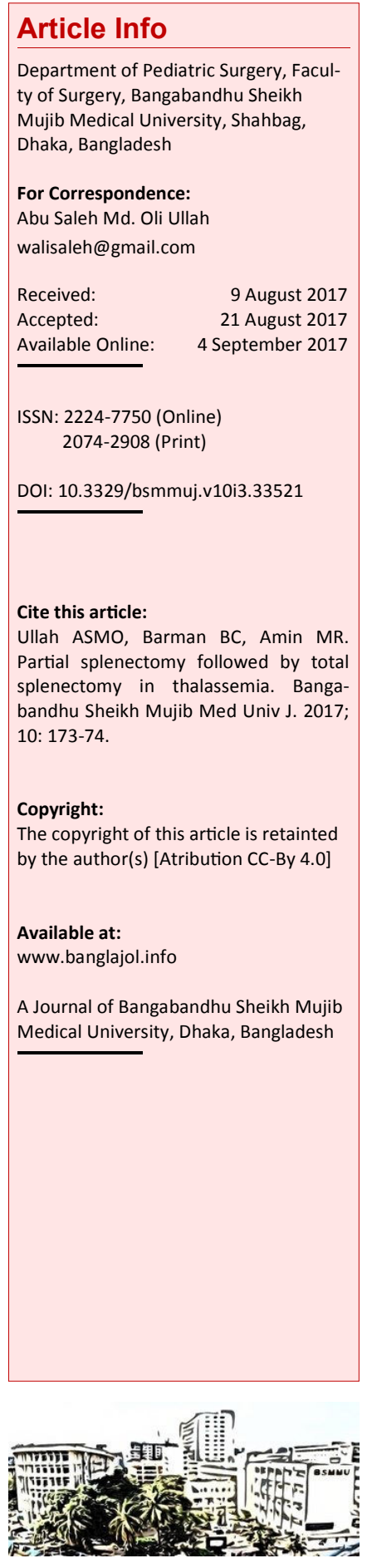

\section{Abstract}

Total splenectomy is the usual operative practice for children with thalassemia requiring splenectomy. But wherever possible, the surgical community should carefully consider partial splenectomy as an alternative treatment option that may offer few important benefits to the patient with the same indication. Here, we are reporting a case where a thalassemic boy enjoyed six years free from overwhelming post-splenectomy infection after partial splenectomy without any vaccination or prophylactic antibiotic before eventual total splenectomy. So, partial splenectopmy may be considered as a preferable initial treatment option for the thalassemic patient with splenomegally requiring splenectomy.

\section{Introduction}

Traditionally, we use to practice total splenectomy, a technically simpler operation, as a palliative measure for children with thalassemia requiring splenectomy. But partial splenectomy is technically much more demanding operation. It has been practiced for the same indication in some centers of the world.1 During the six months follow-up period in a study in children with thalassemia showed favorable initial results.? After six years of partial splenectomy, one boy of this study group presented to us for total splenectomy, at BSMMU, again with increasing need of too much blood transfusions, secondary hypersplenism and mechanical discomfort with huge splenomegaly.

Here, we report this case so that the surgeon community should appreciate carefully the role of partial splenectopmy as a palliative treatment option for the thalassemic patient with splenomegally requiring splenectomy.

\section{Case Report}

At 6 year old boy, a known case of beta thalassemia major since diagnosis at 1.5 years of age (hemoglobin $3.1 \mathrm{gm} / \mathrm{dL} ; \mathrm{HbF} 94.7 \%$ and $\mathrm{HbA}_{2} 5.3 \%$ on electrophoresis; platelet count 203,000/cu-mm, WBC count 7,100/cu-mm; reticulocyte count $11.23 \%$; serum ferritin $154.4 \%$ ), underwent partial splenectomy at BSMMU, (roughly about $125 \mathrm{~mL}$ upper segment of spleen preserved) for increasing need of too much blood transfusions $(745 \mathrm{~mL}$ of whole blood $/ \mathrm{kg} /$ year), secondary hypersple- nism (hemoglobun $4.6 \mathrm{gm} / \mathrm{dL}$, platelet count 95,000/cu-mm, WBC count 3,900/cu-mm); and mechanical discomfort with huge splenomegaly (volume 1,235 mL).

One year after partial splenectomy, his followup study showed: hemoglobin $8.5 \%$; platelet count 235,000/cu-mm; WBC count 9,400/cu$\mathrm{mm}$; his transfusion requirement dropped to $140 \mathrm{~mL}$ of whole blood $/ \mathrm{kg} /$ year; his spleen volume became $292 \mathrm{~mL}$. He was doing well and stopped attending the follow-up clinic thereafter.

After 6 years of partial splenectomy, the boy now 12 years old, again presented to the Department of Pediatric Surgery, BSMMU with recurrent features of huge splenomegaly (volume 1, $560 \mathrm{~mL}$ ), hypersplenism (hemoglobin 5.1 $\mathrm{gm} / \mathrm{dL}$, platelet count 90,000/cu-mm, WBC count 3,900/cu-mm); and increasing need of too much blood transfusions $(850 \mathrm{~mL}$ of whole blood $\mathrm{mL} / \mathrm{kg} /$ year). He was neither immunezed by any pre-operative vaccine against $H$. influenza, Pneumococcus or Meningococcus before partial splenectomy, nor taking any prophylactic antibiotic after partial splenectomy. He did not develop any overwhelming sepsis during the six post partial splenectomy years.

We admitted the boy and did total splenectomy under appropriate vaccination coverage. Like any redo surgery, it was a difficult operation due to intense fibrotic adhesion in the vicinity of spleen. The post-operative period was uneventful. We discharged him on $8^{\text {th }}$ postoperative day with lifelong penicillin prophylaxis. His $7^{\text {th }}$ post-operative day follow-up showed: hemoglobin $9.8 \mathrm{gm} / \mathrm{dL}$, platelet count 
460,000/cu-mm and WBC count 14,000/cu-mm.

\section{Discussion}

A child with beta thalassemia major need regular blood transfusion to maintain hemoglobin level, iron-chelating agent to combat hemosiderosis and eventually splenectomy for prolonging life expectancy and palliation. In thalassemic patients hypersplenism is most probably due to hypertrophy of the reticuloendothelial system. It is suspected before splenomegaly, leucopenia and/or thrombocytopenia, and chiefly by an increase in transfusion requirement to maintain a normal hemoglobin concentration. This result in an increased iron overload in hypertransfused patients. Despite chelation treatment, iron balance is positive in most of these subjects, exposing them to the morbidity and mortality of hemosiderosis, hepatic and endocrine complications and cardiac failure. Splenectomy reduces blood consumption and with desferrioxamine treatment allows iron balance to be achieved. 3 Splenectomy is beneficial against transfusional hemosiderosis even if it suppresses a reticuloendothelial iron store. $\underline{4}$ As palliative procedure, splenectomy is indicated when blood transfusion requirement exceeds $250 \mathrm{~mL} / \mathrm{kg} /$ year, hypersplenism producing splenomegaly with hypocellularity in peripheral blood film, huge splenomegaly causing mechanical discomfort- alone or in combination.

When indicated, total splenectomy is much more commonly practiced traditional operation because of its simplicity and easily controllable fewer peroperative complications. But, total splenectomy also exposes the patient to an important risk of infection: Over-whelming post-splenectomy infections are frequent in children under 5 years but also found in adults. 4 The most commonly involved organisms are Streptococcus pneumoniae (about 50\% of cases), Haemophilus influenzae and Neissenra meningitides.. $\mathbf{5 , 6}$

Per-operatively partial splenectopmy technically demands adequate exposure, complete mobilization of the spleen and tail of the pancreas to the midline, clear anatomical display of the polar and segmental branches of its blood supply, their tedious ligation and division, meticulous hemostasis of the oozing raw surface of the preserved splenic part and even forcing the surgeon to convert to total splenectomy under excessive uncontrolled bleeding in some cases. These require a level of expertise not easily available at centers other than tertiary level hospitals. Nevertheless, partial splenectomy preserves the immunological function of spleen and prevents overwhelming post-splenectomy infection by the above mentioned capsulated bacteria and malarial parasites- which obviates the need for prolonged 'difficult to follow' mandatory antibiotic prophylaxis, inevitably associated with total splenectomy.

This particular boy, now 12 years old, after partial splenectomy, was free from overwhelming sepsis during his six post-partial splenectomy years without any pre-operative vaccination or prophylactic antibiotic. This added 6 quality years to his life before eventual total splenectomy by obviating the need for prolonged and 'difficult to follow' mandatory antibiotic prophylaxis.

Usually indication for partial splenectopmy is limited specially to children under 5 years, whose risk of severe infection is greatest.? But for any age of thalassemic patients requiring splenectomy, partial splenectopmy leaves the option of total splenectomy open- should relapse occur. Thus, Partial splenectopmy as the initial procedure, may add some quality years to thalassemic patients of all ages before eventual total splenectomy by obviating the need for prolonged and 'difficult to follow' mandatory antibiotic prophylaxis.

\section{Conclusion}

Partial splenectopmy may be considered as a preferable initial treatment option for the thalassemic patient with splenomegally requiring splenectomy, specially at tertiary hospitals, where expert technical assistance is not a problem.

\section{References}

1. Montalembert MD, Girot R, Revillon $Y$, Jan $D$, Adjrad L, Ardjoun FZ, Belhani M, Najean Y. Partial splenectomy in homozygous beta thalassaemia. Arch Dis Child. 1990; 65: 304-07.

2. Rahman M. Outcome of partial splenectopmy for hypersplenism in children with thalassemia. MD Thesis, Bangabandhu Sheikh Mujib Medical University, 2012.

3. Graziano JH, Piomelli S, Hilgartner $\mathbf{M}$, et al. Chelation therapy in 1-thalassaemia major III. The role of splenectomy in achieving iron balance. J Pediatr. 1981; 99: 695-99.

4. Modell B. Total management of thalassaemia major. Arch Dis Child. 1977; 52: 489-500.

5. Gelfand JA. Case records of the Massachussets General Hospital. N Engl J Med. 1983; 308: 1212-18.

6. Girot R. Splenectomy in fi thalassaemia. In: Thalassemia today. Sirchia G, Zanella A (eds). The Mediterranean experience. Milano, Centro Transfusionale Ospedale Polyclinico di Milano Editore, 1987, pp 99-104. 